\title{
Weyl Josephson circuits
}

\author{
Valla Fatemi ${ }^{*} *$ \\ Department of Applied Physics, Yale University, New Haven, Connecticut 06520, USA
}

Anton R. Akhmerov (

Kavli Institute of Nanoscience, Delft University of Technology, P.O. Box 4056, 2600 GA Delft, The Netherlands

Landry Bretheau 1 ()

LSI, CEA/DRF/IRAMIS, CNRS, Ecole Polytechnique, Institut Polytechnique de Paris, 91120 Palaiseau, France

(Received 25 September 2020; accepted 2 February 2021; published 29 March 2021)

\begin{abstract}
We introduce Weyl Josephson circuits: small Josephson junction circuits that simulate Weyl band structures. We first formulate a general approach to design circuits that are analogous to Bloch Hamiltonians of a desired dimensionality and symmetry class. We then construct and analyze a six-junction device that produces a threedimensional (3D) Weyl Hamiltonian with broken inversion symmetry and in which topological phase transitions can be triggered in situ. We argue that currently available superconducting circuit technology allows experiments that probe topological properties inaccessible in condensed matter systems.
\end{abstract}

DOI: 10.1103/PhysRevResearch.3.013288

\section{INTRODUCTION}

Topological classification is a building block in our understanding of condensed matter systems [1-3]. Electronic matter that may exhibit topologically nontrivial ground states includes insulators, semimetals, and superconductors. These ideas have been rapidly introduced to many other physical systems, such as quantum circuits [4-6] and metamaterials [7]. We now understand that materials are just one platform in which to explore the physics and potential applications of topologically nontrivial systems.

Topologically protected degeneracies are particular points of interest. Three-dimensional (3D) Weyl bands are an example in which pairs of nodes in the spectrum (e.g., degeneracies of two bands) with opposite topological charges persist over an extended region of Hamiltonian parameter space: A full band gap may open only when nodes of opposite charge have merged or by direct internode scattering. When a material exhibits these bands, it is called a Weyl semimetal $[8,9]$. The peculiar topology of Weyl semimetals results in a host of unusual physical observables, including surface dispersion arcs, strong Berry curvature effects, and responses under interband excitation [10-13]. A menagerie of connected topological band structures with nodal manifolds protected by symmetry have since been proposed and investigated [14-16].

\footnotetext{
*valla.fatemi@yale.edu
}

Published by the American Physical Society under the terms of the Creative Commons Attribution 4.0 International license. Further distribution of this work must maintain attribution to the author(s) and the published article's title, journal citation, and DOI.
However, known Weyl semimetal materials often have additional physics that can obscure phenomena associated with the Weyl nodes (see Ref. [17] for a discussion), and as a result these topological Hamiltonians are being sought out on different platforms [18-22]. In particular, superconductorbased devices and circuits have shown promise for realizing topological states [23], including investigation of topological concepts more generally $[4,5,20,24,25]$ and for the protection of quantum information [26-29]. In 2015, high-transmission multi-terminal superconductor-normal-superconductor (SNS) devices were proposed as a path to emulate Weyl Hamiltonians in the control parameters of fermionic eigenstates [30-35]. Such proposals, however, require nanoscopic control of electronic wave functions within complex nanostructures based on low-dimensional conductors, at the limit of modern technology. Indeed, despite multiple attempts [36-39], no multiterminal SNS structures have exhibited the topological physics of these proposals. Altogether this raises a question: Is an experiment-ready platform well suited to emulate the same physics?

We affirmatively answer this question by expanding the concept to the bosonic collective modes of nonlinear microwave-frequency circuits. For this, we draw on circuits based on linear elements $[18,40,41]$ and standard Josephson tunnel junctions, which are both well developed-the design, fabrication, and measurement of the circuit's nonlinear collective modes are all reliable, standard processes for experimental groups. The robustness of these building-block circuit elements has provided a foundation for the development of ever more complicated qubits and quantum information systems in the past decades. In essence, such circuits offer great flexibility for designing in situ tunable Hamiltonians [42] based on their collective modes. 
Here, we describe an approach to construct small Josephson junction circuits that simulate single-particle Hamiltonians with designable dimensionality and in situ controllable symmetry classes. We describe specific applications that simulate Weyl band structures, including ones that can be tuned through a topological phase transition. We thus dub these Weyl Josephson circuits. The proposed circuits exhibit all the features of Weyl band structures: protected energy degeneracies, divergent Berry curvature near those degeneracies, and the quantized topological invariant. We argue the topologically nontrivial nature of the circuits can be measured in experiments that are unavailable to real materials.

\section{BUILDING THE CIRCUITS}

\section{A. General circuit considerations}

The circuits we consider contain Josephson tunnel junctions as nonlinear inductive elements as well as linear capacitances such as those associated to the tunnel junctions. These circuits have the Hamiltonian [42]

$$
\begin{aligned}
\hat{\mathcal{H}}= & \frac{(2 e)^{2}}{2}\left(\hat{\boldsymbol{n}}-\boldsymbol{n}_{g}\right)^{T} \mathcal{C}^{-1}\left(\hat{\boldsymbol{n}}-\boldsymbol{n}_{g}\right) \\
& -\sum_{i, j} E_{J_{i j}} \cos \left(\hat{\varphi}_{i}-\hat{\varphi}_{j}-\gamma_{i j}\left(\boldsymbol{\varphi}_{m}\right)\right) .
\end{aligned}
$$

Here the capacitive part depends on the number operators $\hat{n}_{i}$ counting the Cooper pairs on each circuit node $i$. The gate voltages offset each charge operator in the capacitive energy by an offset charge $n_{g i}$. For compactness, we use vector notations $\hat{\boldsymbol{n}}, \boldsymbol{n}_{g}$, and an inverse capacitance matrix $\mathcal{C}^{-1}$ that encompasses details of the circuit. The Josephson energy of each junction is $E_{J_{i j}}$, and $\hat{\varphi}_{i}$ are the phase operators canonically conjugate to $\hat{n}_{i}$. The phase offsets $\gamma_{i j}\left(\boldsymbol{\varphi}_{m}\right)$ depend only on the magnetic fluxes $\varphi_{m}$ up to a gauge choice. For convenience, we measure fluxes in units of the superconducting flux quantum $\hbar / 2 e$.

Similar to a Bloch Hamiltonian $\mathcal{H}(\boldsymbol{k})$, the Hamiltonian $\mathcal{H}\left(\boldsymbol{n}_{g}, \boldsymbol{\varphi}_{m}\right)$ of Eq. (1) is periodic in the continuous offset variables, $\boldsymbol{\varphi}_{m}$ and $\boldsymbol{n}_{g}$ (for $\boldsymbol{n}_{g}$ this is true up to an integer translation in $\hat{\boldsymbol{n}})$. Regardless of the device geometry, $\mathcal{H}\left(\boldsymbol{n}_{g}, \boldsymbol{\varphi}_{m}\right)$ satisfies the following symmetry constraints. First, it has a charge inversion symmetry $\mathcal{I} \mathcal{H}\left(\boldsymbol{n}_{g}, \boldsymbol{\varphi}_{m}\right) \mathcal{I}^{-1}=\mathcal{H}\left(-\boldsymbol{n}_{g},-\boldsymbol{\varphi}_{m}\right)$, with the inversion operator $\mathcal{I}=\delta_{n,-n^{\prime}}$ in the charge basis, and $\delta_{n, m}$ is the Kronecker delta. Additionally, the time-reversal symmetry $\mathcal{T}$ reads $\mathcal{T} \mathcal{H}\left(\boldsymbol{n}_{g}, \boldsymbol{\varphi}_{m}\right) \mathcal{T}^{-1}=\mathcal{H}\left(\boldsymbol{n}_{g},-\boldsymbol{\varphi}_{m}\right)$, with the antiunitary operator $\mathcal{T}$ being complex conjugation in the charge basis. Circuits with equal elements may have other unitary symmetries [43] that we leave for later work.

We utilize these symmetry relations to set the symmetry properties of our simulated Hamiltonian. A selection of offset parameters comprise effective crystal momenta $\boldsymbol{k}$ that span the Brillouin zone of the simulated Hamiltonian, with the remainder being control parameters. To emulate a time-reversal symmetric dispersion relation, we choose to vary all magnetic fluxes, while keeping the offset charges constant. Unless $\boldsymbol{n}_{g} \in\{0,1 / 2\}$, this results in a dispersion relation that lacks inversion symmetry. A dual way to realize a time-reversal symmetric dispersion relation is to vary $\boldsymbol{n}_{g}$ while keeping $\boldsymbol{\varphi}_{m}$ constant. Varying all magnetic fluxes and offset charges (a mixed set of parameters $[44,45]$ ) at once corresponds to

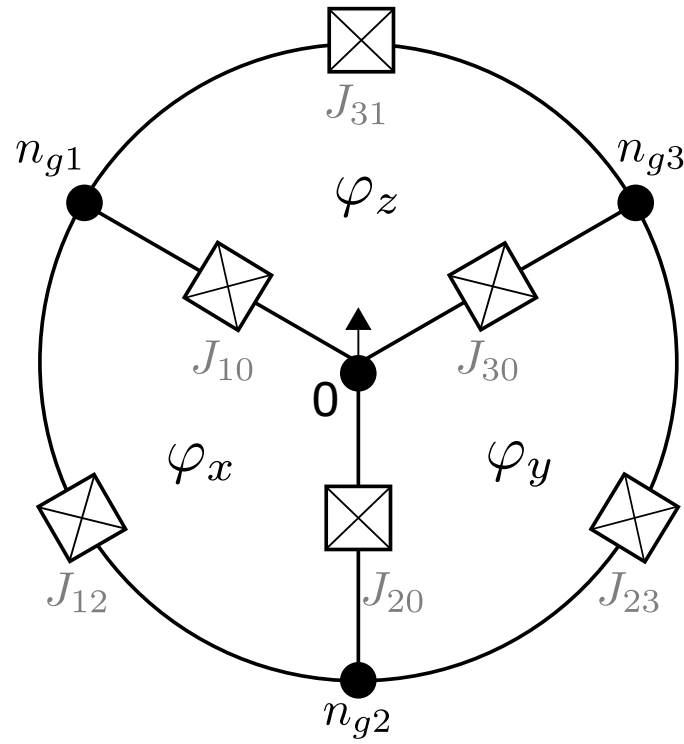

FIG. 1. Diagram of a Weyl Josephson circuit that simulates an inversion-symmetry-breaking Weyl semimetal. Six Josephson tunnel junctions $J_{i j}$ (which include both a capacitance and a Josephson energy) connect nodes $i$ and $j$, where $i=0$ is the reference node. The active nodes $i \in\{1,2,3\}$ are labeled with their gate charge parameter $n_{g i}$, and the three loops are threaded by the reduced magnetic fluxes $\boldsymbol{\varphi}_{m}=\left(\varphi_{x}, \varphi_{y}, \varphi_{z}\right)$, expressed in units of the reduced flux quantum $\hbar / 2 e$.

an inversion-symmetric dispersion relation that lacks timereversal symmetry. Finally, fixing a mixed set of fluxes and offset charges at a value not equal to 0 or $1 / 2$ results in a fully asymmetric dispersion relation.

\section{B. Constructing a time-reversal symmetric Weyl Josephson circuit}

We focus now on the case of a time-reversal symmetric Weyl circuit. Other circuits, with different symmetry classes and dimensions, are discussed in Sec. V. According to the symmetry properties of Josephson circuits, a dispersion relation with Weyl points and a time-reversal symmetry may manifest only in a circuit with at least three independent magnetic fluxes or three offset charges. For convenience, we choose a circuit that satisfies both these requirements in a symmetric way, shown in Fig. 1 [46]. We now choose $\boldsymbol{k}=$ $\boldsymbol{\varphi}_{m}=\left(\varphi_{x}, \varphi_{y}, \varphi_{z}\right) \in[0,2 \pi]^{3}$ and utilize $\boldsymbol{n}_{g}=\left(n_{g 1}, n_{g 2}, n_{g 3}\right) \in$ $[0,1]^{3}$ as control parameters.

We derive the Hamiltonian of this circuit in Appendix A. To obtain its spectrum and eigenstates, we truncate the Hilbert space to contain several lowest energy charge states and numerically diagonalize the Hamiltonian projected on this subspace. To present the generic physics more transparently, we take all Josephson energies as well as capacitive energies to be equal and consider the charge-dominated regime, $E_{C} \gg E_{J}$, with $E_{C}=(2 e)^{2} / 2 C$ being the charging energy of a single junction. In this situation, the minimal configuration of interest is in the vicinity of four charge states tuned to similar energy via the gate charges $\boldsymbol{n}_{g}$. For example, choosing the globally uncharged state $\left|n_{1} n_{2} n_{3}\right\rangle=|000\rangle$ (all 
active nodes have zero net charge) and all the singly charged states $\{|100\rangle,|010\rangle,|001\rangle\}$ (a single Cooper pair on any one of the three islands), we obtain the following simplified Hamiltonian:

$$
\hat{\mathcal{H}}\left(\boldsymbol{\varphi}_{m}, \boldsymbol{n}_{g}\right)=-\frac{E_{J}}{2}\left(\begin{array}{cccc}
-\lambda\left(n_{g 1}+n_{g 2}+n_{g 3}\right) & 1 & 1 & 1 \\
1 & -\lambda\left(1-n_{g 1}\right) & e^{i \varphi_{x}} & e^{-i \varphi_{z}} \\
1 & e^{-i \varphi_{x}} & -\lambda\left(1-n_{g 2}\right) & e^{i \varphi_{y}} \\
1 & e^{i \varphi_{z}} & e^{-i \varphi_{y}} & -\lambda\left(1-n_{g 3}\right)
\end{array}\right),
$$

where $\lambda=E_{C} / E_{J} \gg 1$. All four charge states are approximately degenerate at $\boldsymbol{n}_{g 0}=\frac{1}{4} \mathbf{1}$, where $\mathbf{1}=(1,1,1)$ is the unit diagonal vector. Thus, when $\lambda\left\|\boldsymbol{n}_{g}-\boldsymbol{n}_{g 0}\right\|<1$, flux bias has a significant effect on the system. With this Hamiltonian in hand, we now turn to an inspection of its physics.

\section{TOPOLOGICAL SPECTRUM AND INVARIANTS}

\section{A. Energy spectrum}

We first inspect the dispersion relation of the Hamiltonian (2). When the charge states are electrostatically degenerate $\left(\boldsymbol{n}_{g}=\boldsymbol{n}_{g 0}\right)$, the ground state is doubly degenerate at two points $\boldsymbol{\varphi}_{m}=\frac{\pi}{2} \mathbf{1}, \frac{3 \pi}{2} \mathbf{1}$, with $\mathbf{1}=(1,1,1)$, and triply degenerate at the point $\boldsymbol{\varphi}_{m}=\pi \mathbf{1}$, as shown in Fig. 2(a) [47]. These nodes lie along the major diagonal of the Brillouin zone $\varphi_{m}=\varphi_{\text {diag }} 1$ due to the spatial symmetry of the circuit.

Varying the gate charge induces a topological phase transition. We consider uniform gating, $\boldsymbol{n}_{g}=\boldsymbol{n}_{g 0}+n_{\text {diag }} \mathbf{1}$ with $0<n_{\text {diag }}<0.25$, which retains the symmetry that leaves all nodes on the major diagonal of the Brillouin zone. As shown in Figs. 2(b)-2(e), the triply degenerate point splits into two doubly degenerate points while still lying along the major diagonal of flux. At a certain point, $n_{\text {diag }}-1 / 4 \approx-0.13 \lambda^{-1}$, the Weyl nodes converge and annihilate each other, signaling the topological phase transition. Beyond this point, the ground state is gapped from the higher energy manifold for all flux configurations.

The Weyl nodes also survive asymmetric tuning of $\boldsymbol{n}_{g}$ and nonuniformity of the Josephson energies. This can, for example, induce the Weyl nodes to shift off of the major flux diagonal. Figure 3(a) shows these effects for a configuration with no particular symmetry except the time-reversal symmetry guaranteed by the choice of effective crystal momenta. The Weyl node locations are indicated by the spheres (the meaning of the color, the topological charge, will be described in the next subsection). While offset charge detuning can generally induce the topological transition, the necessary amount of such detuning depends on the particular parameters of the device, particularly $E_{J} / E_{C}$ (see Appendix B 1).

\section{B. Quantum geometry and topological invariants}

We now investigate topological aspects of the ground-state wave function, which is accomplished by inspecting its Berry curvature $\boldsymbol{\Omega}$. The Berry curvature relates the overlap between derivatives of the wave function $|\Psi\rangle$ with respect to different Hamiltonian parameters, as in Eq. (3):

$$
\begin{gathered}
\boldsymbol{\Omega}=\operatorname{Im} \sum_{i, j, k}\left\langle\partial_{\varphi_{i}} \Psi \| \partial_{\varphi_{j}} \Psi\right\rangle \epsilon_{i j k} \boldsymbol{e}_{k}, \\
C\left[S\left(\boldsymbol{\varphi}_{m}\right)\right]=\frac{1}{2 \pi} \oint_{S} \boldsymbol{d} \boldsymbol{S} \cdot \boldsymbol{\Omega},
\end{gathered}
$$
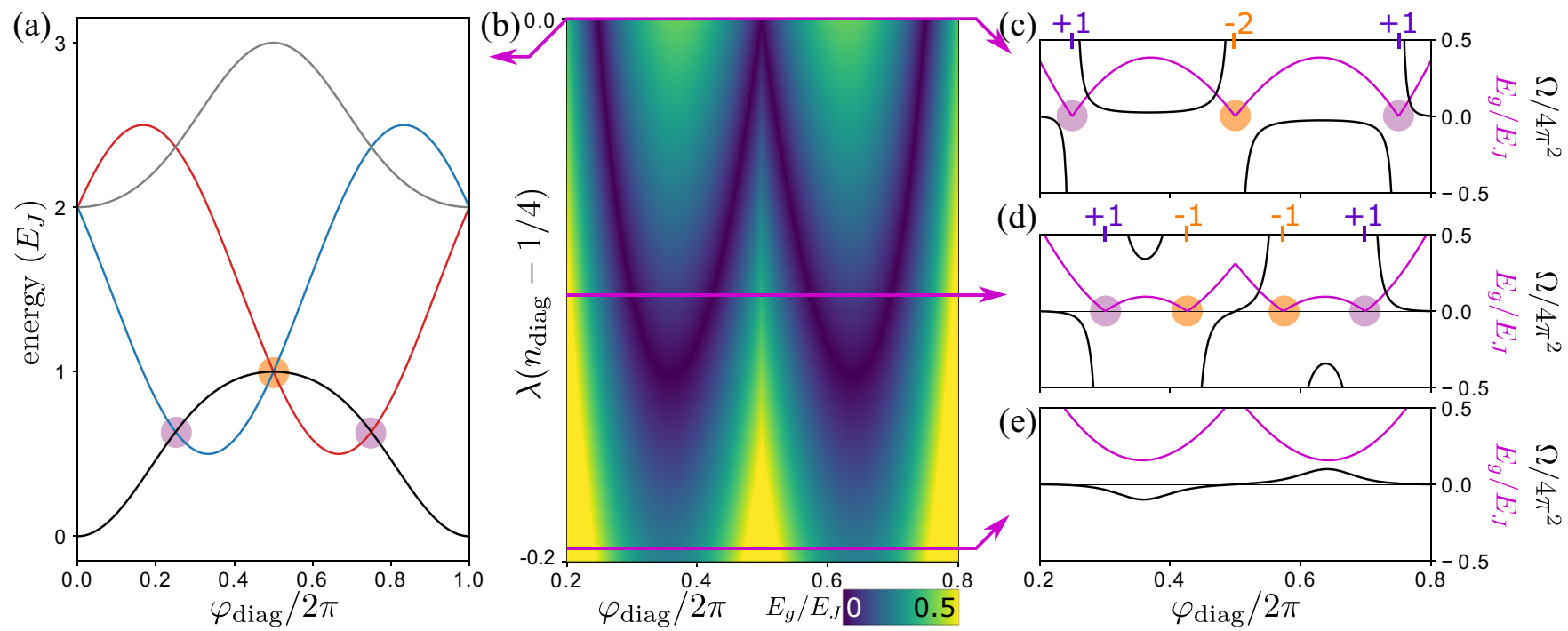

FIG. 2. (a) Energy spectrum in the charge-dominated regime from (2) at $\boldsymbol{n}_{g}=\boldsymbol{n}_{g 0}$ as a function of flux along the main diagonal $\varphi_{\text {diag }}$. Line color is determined from wave-function continuity [48]. (b) Excitation gap from the ground state to the first excited state ( $\left.E_{g}\right)$ as a function of symmetric gate charge $\boldsymbol{n}_{g}-\boldsymbol{n}_{g 0}=n_{\text {diag }} \mathbf{1}$ and $\varphi_{\text {diag. }}$. [(c)-(e)] Line cuts of the excitation gap $E_{g}$ (pink) and the Berry curvature $\Omega$ (black) along $\varphi_{\text {diag }}$ for the indicated gate charge values. 

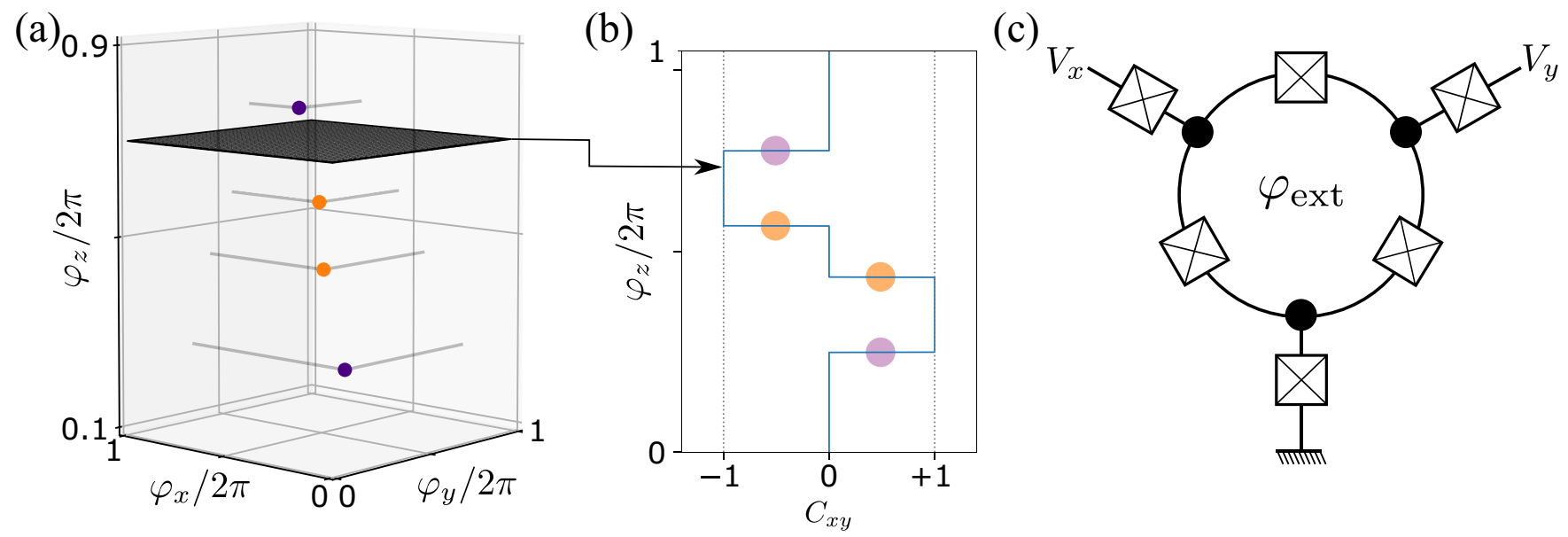

FIG. 3. (a) Location of the Weyl points in flux space for $\lambda n_{\text {diag }}=0.08$ and $E_{J_{12}}=1.7 E_{J}$. Like-charged nodes exist at opposite momenta, as in an inversion-symmetry broken Weyl semimetal. The gray plane indicates the integration area that determines the Chern number $C_{x y}$ for $\varphi_{z}=$ $0.7 \times 2 \pi$. (b) Chern number as a function of $\varphi_{z}, C_{x y}\left(\varphi_{z}\right)$, for the same parameters as in panel (a). (c) Broken-open circuit for transconductance measurements. The upper leads are biased by dc voltages $V_{x}$ and $V_{y}$ referenced to the grounded lower lead, and the loop is threaded by a flux $\varphi_{\text {ext }}$. Measurement of quantized dc transconductance would reveal the Chern number [32].

where $\epsilon_{i j k}$ is the Levi-Civita symbol and $\boldsymbol{e}_{k}$ is the unit vector in direction $k$. Because of its closeness to a second derivative of the wave function, it is referred to as a curvature and described as a quantum geometry. Its integral over a surface $S\left(\boldsymbol{\varphi}_{m}\right)$ enclosing a Weyl point [Eq. (4)] is a Chern number $C \in \mathbb{Z}$ that determines the topological charge of the Weyl point. Nonzero Berry curvature and Chern number both are associated with physical observables, which will be described in Sec. IV.

The Berry curvature of the ground state is plotted alongside the energy gap in Figs. 2(c)-2(e). In the topological phase [Figs. 2(c) and 2(d)], the Berry curvature diverges where the ground and excited states are degenerate. Integrating the Berry curvature around each node [Eq. (4)], we determine the topological charges indicated in Figs. 2(c) and 2(d). The topological phase transition occurs when nodes of opposite charge converge and annihilate each other, as can be seen in the transition from Figs. 2(d) to 2(e).

There are four distinct Weyl points in most of the topologically nontrivial situations described here, which we calculated to have integer Chern numbers indicated in Figs. 2(c) and 2(d). Doubly charged points may also exist, such as in Figs. 2(a) and 2(c). Crucially, like-charged nodes exist at opposite crystal momenta $C\left[S\left(\boldsymbol{\varphi}_{m}\right)\right]=C\left[S\left(-\boldsymbol{\varphi}_{m}\right)\right]$. This observation remains true in circuits with nonuniform Josephson elements, such as that shown in Fig. 3(a), where the position and charge of the four nodes are indicated by colored spheres. This establishes that this circuit is a simulator of a broken-inversion-symmetry Weyl semimetal with preserved time-reversal symmetry [49], as expected from the symmetrybased design.

\section{EXPERIMENTAL OBSERVABLES}

In the previous sections, we outlined the principles of construction, the resulting spectrum, and abstract topological aspects of a Weyl Josephson circuit. In this section, we describe three experimental observables to probe the topologically nontrivial character: transition spectra, adiabatic responses measuring Chern number, and nonadiabatic responses measuring Berry curvature. The latter two both rely on the consequences of Berry curvature on the system response function [50]. In regards to circuit parameters and noise robustness, all these experiments are accessible using modern nanofabrication and measurement techniques (see Appendix B for details). Some of these experiments are not possible to perform on a real material, highlighting the complementary nature of parametric simulation with superconducting quantum circuits.

\section{A. Microwave spectroscopy of topological phase transitions}

The simplest experiment is microwave spectroscopy of the circuit under otherwise static conditions. The goal is to measure the energy spectrum as a function of $\boldsymbol{k}$ and detect degenerate points. The smoking gun is to observe that these Weyl nodes survive when varying the gate charges over a finite range and finally annihilate following a topological phase transition. In practice, one needs to perform such spectroscopy within $\approx[0,50] \mathrm{GHz}$ (the exact range will depend on the particular circuit parameters chosen). One possibility is to perform the standard two-tone spectroscopy used in circuit QED by coupling the circuit to a superconducting resonator [51-54]. Another approach is Josephson spectroscopy, which uses a voltage-biased Josephson tunnel junction as an on-chip microwave spectrometer [55-59]. These two methods are complementary since Josephson spectroscopy is better suited for high frequencies (typically within [2,100] $\mathrm{GHz}$ ) while two-tone spectroscopy works better at lower frequencies (typically within $[0.1,30] \mathrm{GHz}$ ).

\section{B. Transconductance to measure Chern number}

A direct measurement of topological invariants is a stronger indication of a topologically nontrivial state. The Chern number may be accessed by measuring the response due to adiabatic variation of Hamiltonian parameters, which 
can be accomplished by transconductance measurements [32]. The periodicity of the Hamiltonian means two-dimensional (2D) planes in flux space bounded by the effective Brillouin zone constitute closed manifolds and therefore have a Chern number. Figure 3(b) shows the Chern number $C_{x y}\left(\varphi_{z}\right)$ for the $\left(\varphi_{x}, \varphi_{y}\right)$ plane as a function of $\varphi_{z}$ (now treated as a control knob), for the circuit in a topological phase. Whenever a Weyl node is crossed by tuning $\varphi_{z}$ [see Fig. 3(a)], the Chern number changes by an integer amount corresponding to the charge of that node. After a topological phase transition into a trivial phase, the Chern number is zero for any value of $\varphi_{z}$. These Chern numbers can be probed by performing transconductance measurements, as proposed by Riwar and collaborators for the case of multiterminal high-transmission Josephson junctions [32]. Similarly, here one may break open two of the loops of the Weyl circuit and apply dc voltages [see Fig. 3(c)]. Using the ac Josephson effect, two of the fluxes can be varied adiabatically and linearly with time while keeping the third flux static. If the rate of change of these two fluxes is incommensurate, then one can sample a complete $2 \mathrm{D}$ plane within the Brillouin zone. This will lead to a dc current that is directly proportional to the Chern number, and therefore to a quantized transconductance $[32,60]$ without the complications of a nearby continuum $[33,61]$. We note that this transconductance has a close relationship with Cooper pair pumps $[4,62,63]$ and may be useful for metrological applications $[63,64]$.

\section{Direct Berry curvature measurements}

Going further, the Berry curvature can be directly measured as a function of the quasimomenta. Then, by simple integration, one could extract the Weyl nodes' topological charge. This approach is based on a theoretical breakthrough describing how Berry curvature can be observed from the nonadiabatic response of physical observables to the rate of change of an external parameter $[50,65]$. This method was recently implemented in the field of quantum circuits using a basic system, a driven qubit, to observe topological phase transitions $[5,66]$. Therefore, our proposed experiment is feasible albeit more involved than the ones discussed before. Indeed, a direct Berry curvature measurement involves coherent manipulation of the circuit's quantum states (superposition of ground and excited states) and therefore requires that the circuit exhibits sufficiently long coherence times ( $\gtrsim 1 \mu \mathrm{s})$. A related approach is to use carefully designed absorption spectroscopy measurements relying on the same underlying physics [35].

\section{ADDITIONAL TOPOLOGICAL JOSEPHSON CIRCUITS}

Finally, we demonstrate the extensibility of our platform by briefly describing three additional circuits that simulate topological band structures in different symmetry classes and dimensions.

\section{A. Minimal inversion symmetric Weyl circuits}

An inversion symmetric Weyl semimetal requires three offset variables, with both types present, to comprise $\boldsymbol{k}$. Minimal configurations satisfying this are drawn in Figs. 4(a) and 4(b). Both have exactly three available offset parameters: The flux (a)
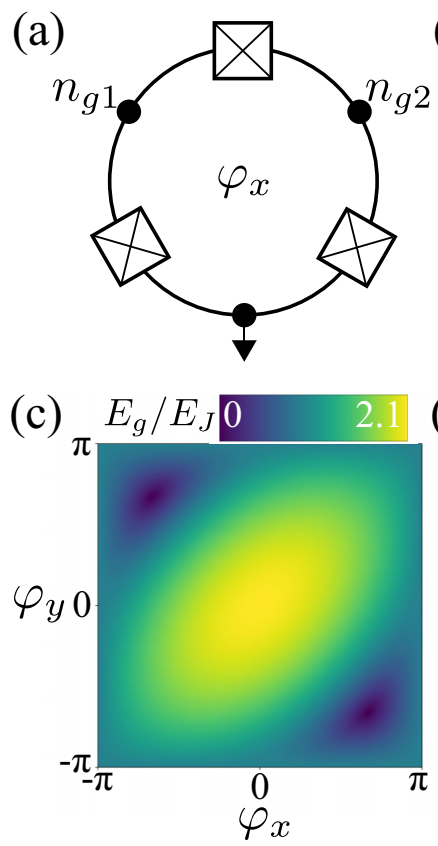

(b)

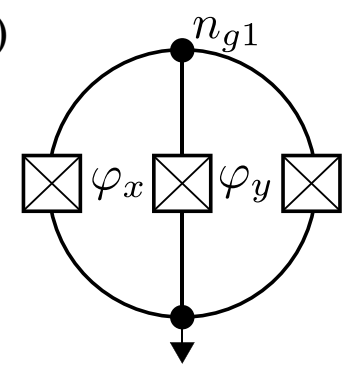

(d)

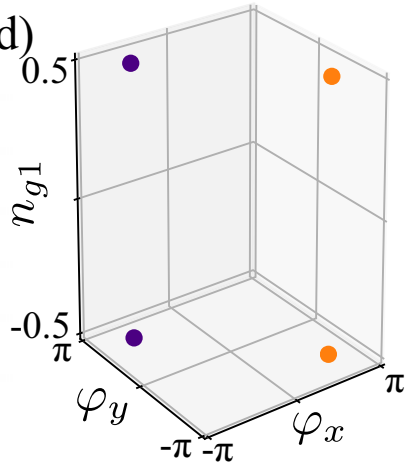

FIG. 4. Minimal inversion-symmetric Weyl Josephson circuits. (a) Flux qubit circuit diagram. (b) Gradiometric-SQUID Cooper pair box circuit diagram. (c) Excitation gap $E_{g}$ of the gradiometricSQUID Cooper pair box as a function of the two fluxes. Here, $n_{g 1}=0.5$ and $E_{J}=E_{C}$ (all junctions identical). (d) Location and charge (purple: +1 , orange: -1 ) of the Weyl nodes. Note: the pairs of nodes located at the top and bottom are the same due to periodicity.

qubit has $\left(n_{g 1}, n_{g 2}, \varphi_{x}\right)$ and the gradiometric-superconducting quantum interference device (SQUID) Cooper pair box has $\left(n_{g 1}, \varphi_{x}, \varphi_{y}\right)$. We focus on the gradiometric-SQUID Cooper pair box, as degeneracies in the flux qubit case have been predicted previously [4]. In Fig. 4(c), we plot the energy gap as a function of the two fluxes for $n_{g 1}=0.5$, where the location of the two nodes in the spectrum are apparent. The nodes have opposite topological charge, as shown in Fig. 4(d). Note that $n_{g 1}= \pm 0.5$ is the Brillouin zone boundary and so only two nodes are present, one of each charge. Nodes of opposite charge are located at opposite quasimomenta, confirming that the circuit simulates an inversion symmetric Weyl band structure [49].

The simplicity of these circuits is attractive for initial experiments. Indeed, the microwave spectroscopy and Berry curvature experiments proposed in Sec. IV are relatively straightforward to consider. An equivalent to the transconductance experiment, however, would require linear variation of $n_{g}$ (e.g., a dc current across a capacitor) in order to span a plane with a finite Chern number.

\section{B. Nodal line circuits in 3D and 4D}

We present here nodal line circuits of two flavors. The first, shown in Fig. 5(a), emulates a nodal-line semimetal of the codimension type, which requires both inversion and time-reversal symmetry (see Ref. [17] for an overview). Here, $\boldsymbol{k}=\left(\varphi_{x}, \varphi_{y}, \varphi_{z}\right)$, while $n_{g 1}$ is a control parameter, which means the circuit generally satisfies the time-reversal symmetry condition. When charge inversion symmetry is restored at 
(a)
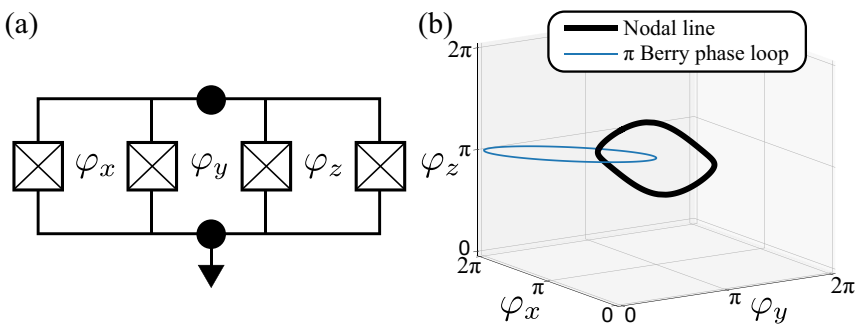

(c)

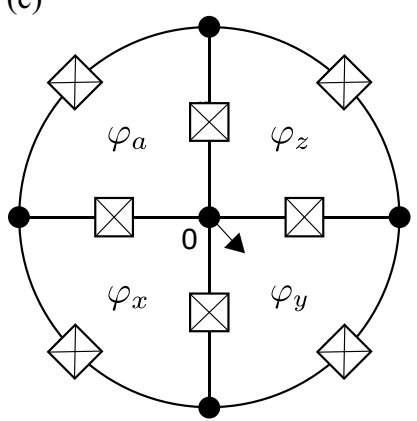

(d)

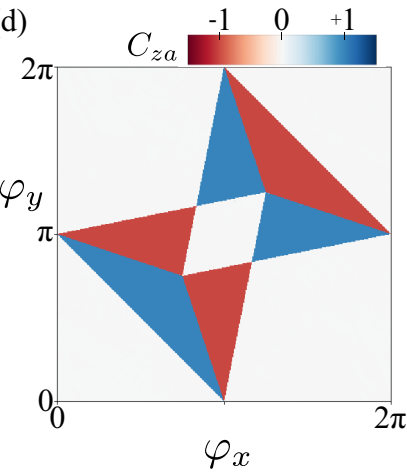

FIG. 5. Nodal line circuits (both calculations $E_{J} \ll E_{C}$ ). (a) Circuit that simulates a nodal line semimetal for $\boldsymbol{k}=\left(\varphi_{x}, \varphi_{y}, \varphi_{z}\right)$ and $n_{g 1}=0.5$. (b) The loop-shaped nodal line encircling $\boldsymbol{k}=(\pi, \pi, \pi)$ (one junction has double the Josephson energy of each of the other three). In blue, we depict an example trajectory with a $\pi$ Berry phase. (c) Circuit that emulates a 4D Weyl band structure $\boldsymbol{k}=$ $\left(\varphi_{x}, \varphi_{y}, \varphi_{z}, \varphi_{a}\right)$ with $1 \mathrm{D}$ Weyl lines. (d) Signatures of the 1D Weyl lines are exhibited by the Chern number in the $\varphi_{z}, \varphi_{a}$ plane as a function of $\varphi_{x}$ and $\varphi_{y}$ (identical junctions).

$n_{g 1}=0.5$, nodal lines may be observed. In Fig. 5(b), we show the momenta of the loop-shaped line degeneracy between the ground and first excited states. Although Berry curvature is zero due to symmetry, a Berry phase of $\pi$ exists for closed trajectories that pass through the nodal loop.

The general approach to circuit construction in Sec. II may be applied to create band structures of higher dimensionality than is possible with actual crystals. Here, we describe a generalization of time-reversal symmetric Weyl band structure in four dimensions [32], where line degeneracies (rather than point degeneracies) are sources of Berry curvature. We choose a circuit with four nodes and four loops, shown in Fig. 5(c), where the choice $\boldsymbol{k}=\boldsymbol{\varphi}_{m}$ ensures time-reversal symmetry. We fix the offset charges so that all five charge states (in the deep charging regime) are degenerate prior to introduction of the Josephson energies, breaking inversion symmetry. To visualize the topological characteristics of this band structure, we compute the Chern number in a $2 \mathrm{D}$ plane $\left(\varphi_{z}, \varphi_{a}\right)$ as a function of the two remaining phases, as shown in Fig. 5(d). Note that expanding to $N$ dimensions in this structure is straightforward by adding additional "wedges" to the circuit.

\section{CONCLUDING REMARKS}

In this paper, we have described a proposal for Weyl Josephson circuits: small Josephson tunnel junction circuits that exhibit topological band structures in the parametric dependence of their collective modes, including in situ triggerable topological phase transitions. We have also described several experiments that probe the topological nature of the circuit. All the necessary ingredients for an experimental implementation are in reach with modern nanofabrication and experimental techniques.

This work leaves open questions on how far these ideas can be developed. An immediate step is the classification of the available symmetry classes, including point group symmetries beyond inversion. An important question to this end is whether we can create robust analogies to spins and spin-orbit coupling in order to create analogs to quantum spin Hall insulators [67]. What may be gained by replacing tunnel junctions with different two-terminal Josephson elements, such as those with high transparency or Majorana junctions? Can small Josephson circuits simulate topological boundaries and their unique surface dispersions? Finally, applying strong driving or dissipation, a standard tool in superconducting circuits, extends the system to symmetry classes that are hard to access in condensed matter systems $[68,69]$. The interplay of Floquet physics with topological ground states [70] and circuit Hamiltonians [71] are active areas of study that can be combined in circuits like the one presented here.

These circuits exhibit clear parallels with theoretical proposals based on microscopic fermionic Andreev states in a scattering region contacted by multiple trivial superconducting leads [31-33,35,60,61] or topological superconducting leads [45,72-75], including cases with nodal lines [76]. It will be interesting to inspect whether such proposals generically have a suitable Josephson circuit analog, particularly when restricting to the even-parity sector and without spin-orbit coupling.

We are convinced that Weyl Josephson circuits offer a versatile, tunable, and complementary platform to probe the physics of topologically nontrivial systems. We hope that this work will stimulate mutually beneficial contact between the fields of superconducting quantum circuits and topological condensed matter physics.

\section{ACKNOWLEDGMENTS}

We thank L. Peyruchat, J. Griesmar, J.-D. Pillet, and Ç. Ö. Girit for fruitful discussions and for sharing their related forthcoming work [63]. We also acknowledge helpful discussions with M. Devoret, N. Frattini, L. Glazman, P. Kurilovich, V. Kurilovich, K. Serniak, C. Smith, and U. Vool; L. Shi and J. Song; and M. Houzet and J. Meyer. We thank A. Eickbusch for assistance regarding the topological volume fraction calculations. L.B. acknowledges support of Agence Nationale de la Recherche through Grant No. ANR-18-CE47-0012 (JCJC QIPHSC). Code and data available on Zenodo [77].

V.F. conceived the idea and developed the theory with guidance from A.A. and L.B. V.F., A.A., and L.B. all wrote the manuscript.

\section{APPENDIX A: SIX-JUNCTION CIRCUIT HAMILTONIAN}

\section{Derivation}

Here we derive the Hamiltonian of the circuit in Fig. 1 via network analysis in the usual way by starting in the nodal flux basis [42]. Using the reduced flux quantum $\phi_{0}=\hbar / 2 e$ to rescale into a phase basis, the Lagrangian is $\mathcal{L}=\mathcal{L}_{C}-U_{J}$, 
where the Josephson part can be written

$$
\begin{aligned}
-U_{J}\left(\boldsymbol{\varphi}, \boldsymbol{\varphi}_{m}\right)= & E_{J_{10}} \cos \varphi_{1}+E_{J_{20}} \cos \varphi_{2}+E_{J_{30}} \cos \varphi_{3} \\
& +E_{J_{12}} \cos \left(\varphi_{1}-\varphi_{2}-\varphi_{x}\right) \\
& +E_{J_{23}} \cos \left(\varphi_{2}-\varphi_{3}-\varphi_{y}\right) \\
& +E_{J_{31}} \cos \left(\varphi_{3}-\varphi_{1}-\varphi_{z}\right)
\end{aligned}
$$

with $\boldsymbol{\varphi}=\left(\varphi_{1}, \varphi_{2}, \varphi_{3}\right)$ and $\boldsymbol{\varphi}_{m}=\left(\varphi_{x}, \varphi_{y}, \varphi_{z}\right)$. The charging part of the Lagrangian reads

$$
\begin{aligned}
\mathcal{L}_{C}= & \frac{\phi_{0}^{2}}{2}\left(C_{10} \dot{\varphi}_{1}^{2}+C_{20} \dot{\varphi}_{2}^{2}+C_{30} \dot{\varphi}_{3}^{2}+C_{12}\left(\dot{\varphi}_{1}-\dot{\varphi}_{2}\right)^{2}\right. \\
& \left.+C_{23}\left(\dot{\varphi}_{2}-\dot{\varphi}_{3}\right)^{2}+C_{31}\left(\dot{\varphi}_{3}-\dot{\varphi}_{1}\right)^{2}\right) \\
= & \frac{\phi_{0}^{2}}{2} \dot{\varphi}^{T} \mathcal{C} \dot{\varphi}
\end{aligned}
$$

where we introduce a convenient $3 \times 3$ capacitance matrix $\mathcal{C}$. For compactness, we ignore constant terms in the Lagrangian and leave off the reference phase defined at the central node $\varphi_{0}$.

We next account for offset charge on each node (which may be controlled by capacitive gates that are formally defined here to have sufficiently small capacitance so as not to impact the circuit modes) and define canonically conjugate momenta in the usual way in order to come to a Hamiltonian:

$$
\hat{\mathcal{H}}=\frac{4 e^{2}}{2}\left(\hat{\boldsymbol{n}}-\boldsymbol{n}_{g}\right)^{T} \mathcal{C}^{-1}\left(\hat{\boldsymbol{n}}-\boldsymbol{n}_{g}\right)+U_{J},
$$

where $\hat{\boldsymbol{n}}=\left(\hat{n}_{1}, \hat{n}_{2}, \hat{n}_{3}\right), \quad \boldsymbol{n}_{g}=\left(n_{g 1}, n_{g 2}, n_{g 3}\right), \quad$ and $\hat{\boldsymbol{\varphi}}=$ $\left(\hat{\varphi}_{1}, \hat{\varphi}_{2}, \hat{\varphi}_{3}\right)$. Finally, it is convenient to choose a characteristic scale for the capacitances in order to write the charge term with a prefactor with units of energy. For simplicity, we choose the average junction capacitance $\bar{C}$, which defines both the characteristic charging energy $E_{C}=(2 e)^{2} / 2 \bar{C}$ and the dimensionless inverse capacitance matrix $c^{-1}=\bar{C} \mathcal{C}^{-1}$ :

$$
\hat{\mathcal{H}}=E_{C}\left(\hat{\boldsymbol{n}}-\boldsymbol{n}_{g}\right)^{T} c^{-1}\left(\hat{\boldsymbol{n}}-\boldsymbol{n}_{g}\right)+U_{J}
$$

\section{Tight-binding model}

We now take advantage of the fact that the individual Josephson terms are equivalently represented as a sum of single-Cooper-pair translation operators:

$$
\begin{aligned}
& E_{J_{i j}} \cos \left[\hat{\varphi}_{i}-\hat{\varphi}_{j}+\gamma_{i j}\left(\boldsymbol{\varphi}_{m}\right)\right] \\
& \quad=\frac{1}{2} E_{J_{i j}} e^{i \gamma_{i j}\left(\boldsymbol{\varphi}_{m}\right)}\left|n_{i}, n_{j}+1\right\rangle\left\langle n_{i}+1, n_{j}\right|+\text { H.c. }
\end{aligned}
$$

These become the hopping terms of the tight-binding model. In the charge-dominated regime, $E_{C} \gg E_{J_{i j}}$, the case of interest studied in the main text is in the vicinity of a four-fold charge degeneracy point, which is in principle analytically soluble. After assuming all $E_{J_{i j}}=E_{J}$, we have the $4 \times 4$ Hamiltonian matrix in Eq. (2). When more than four charge states must be considered, or outside the deep charging regime, the model must be solved numerically with more charge basis states. For this, we employ the Kwant tight binding package [79].

\section{APPENDIX B: EXPERIMENTAL CONSIDERATIONS}

Here we investigate three crucial experimental considerations, focusing on the time-reversal symmetric Weyl circuit that is the focus of the main text.

\section{Beyond deep charging limit}

In the main text, for illustrative purposes, we focus on a simplified Hamiltonian that provides a good approximation of the circuit in the deep charging regime $\left(E_{J} / E_{C} \ll 1\right)$ near $\boldsymbol{n}_{g}=\boldsymbol{n}_{g 0}=\frac{1}{4} \mathbf{1}$, with $\mathbf{1}=(1,1,1)$. However, the regimes in which Josephson energies $E_{J}$ are comparable to or larger than the charging energies $E_{C}$ are important because they are easily accessible in experiment [80,81]. The limit $E_{J} \gg E_{C}$ additionally provides exponentially suppressed sensitivity to offset charge noise in simple circuits like the transmon [81]. We cannot strictly retain this feature in Weyl Josephson circuits due to the topological phase transitions that can be triggered by offset charge tuning.

Nonetheless, choosing $E_{J}$ comparable to $E_{C}$ provides several experimental advantages for topological physics. First, for $E_{J} \approx E_{C} / 2$ the volume fraction of the $\boldsymbol{n}_{g}$ parameter space in which the circuit exhibits ground-state degeneracies in the Brillouin zone is maximized to about 0.27 [Fig. 6(a)]. Maximizing this quantity is advantageous for experiments that must search for the topological regime by varying gate voltages on the islands. Second, the positions of the Weyl nodes become less sensitive to offset charge offsets [Figs. 6(b)-6(e)], an advantage for experiments that may have moderate offset charge drift (see Appendix B 3 for more on this point). Note that for $E_{J} \gg E_{C}$ the system is dominated by wells in the classical Josephson potential-thus, degeneracy points indicate transitions to a new global minimum in which the lowest energy states of the two wells have no avoided crossing. Third, the characteristic energy scale in the topological regime becomes sensitive to $E_{C}$, as indicated in Figs. 6(b)-6(e). This is convenient as $E_{C}$ can lowered by geometric circuit features independent of the junction's intrinsic capacitance. All of these features make the moderate $E_{J} / E_{C}$ regime attractive for experiments. Curiously, as is visible in Figs. 6(b)-6(e), the energy gap along contours connecting Weyl nodes varies with offset charge detuning despite the fact that the position of the nodes changes quite slowly. When this gap approaches zero, the nodes rapidly converge and annihilate. We note that a previous work on Cooper pair pumps [equivalent circuit to Fig. 1(a)] investigated the effect of noise on pumping processes and found that $E_{J} \sim E_{C}$ was optimal for that circuit for different reasons than these [82].

\section{Junction disorder}

Fabrication of Josephson tunnel junctions with aluminum electrodes and $\mathrm{AlOx}$ tunnel barriers is a relatively mature process. Junctions that are fabricated simultaneously and placed in the same region of a wafer can be made identical to within about $2 \%$ accuracy [83]. While the topological nature of the Weyl points implies a general robustness to variations in Hamiltonian parameters, it is reasonable to specifically ask whether a Weyl circuit is robust to the experimental degree of uncertainty. To this end, we model the six-junction circuit 

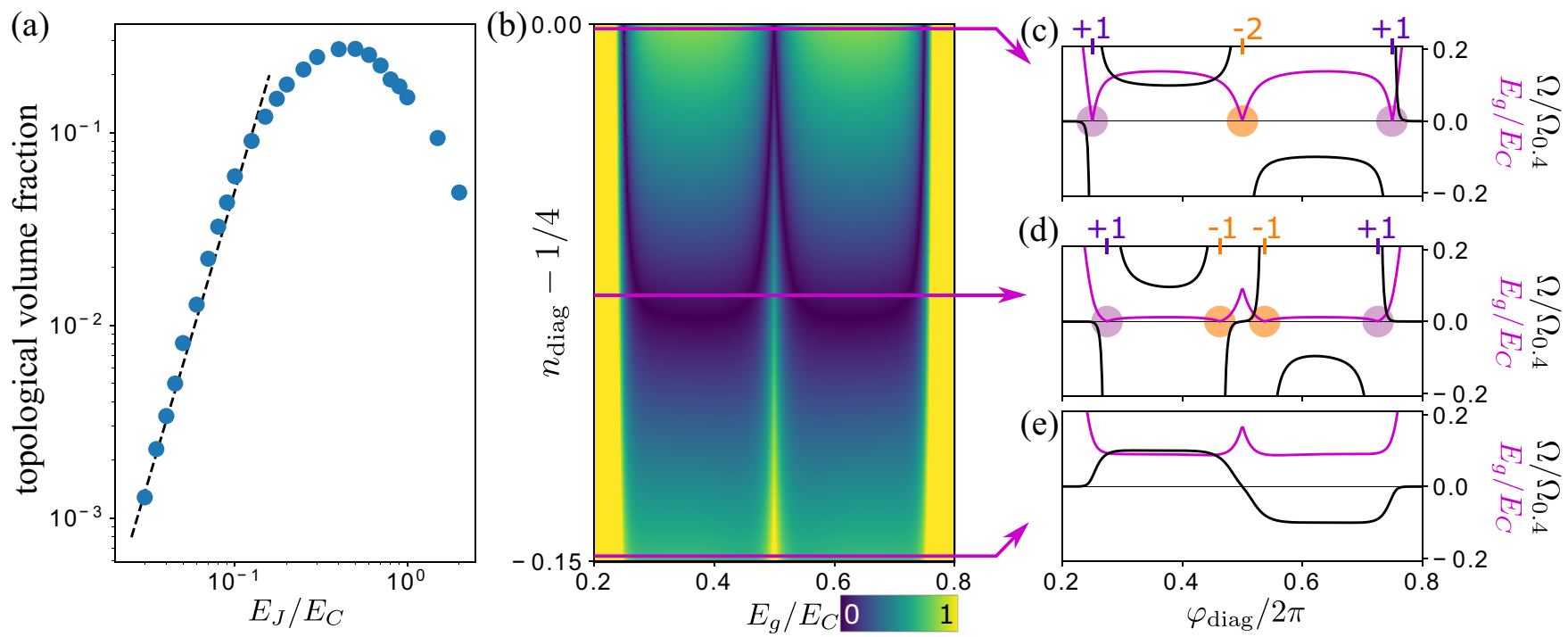

(d)
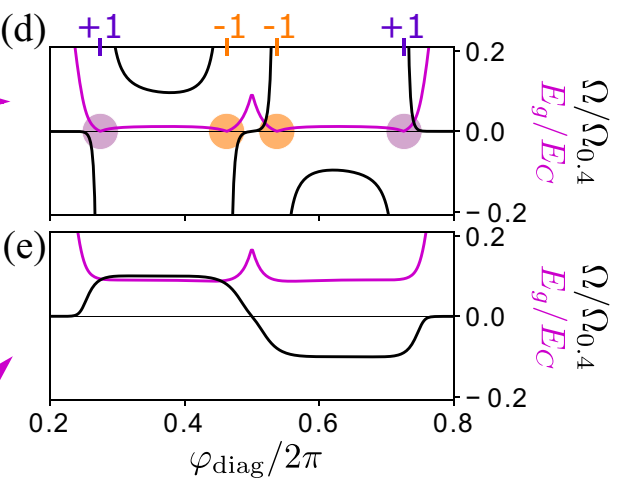

FIG. 6. (a) Topological volume fraction of the $\boldsymbol{n}_{g}$ parameter space as a function of $E_{J} / E_{C}$. This is estimated by finding ground-state degeneracies in $\boldsymbol{\varphi}_{m}$ space on a $50 \times 50 \times 50$ grid spanning the $\boldsymbol{n}_{g}$ cube. The dashed black line indicates a cubic trend. (b) Excitation gap from the ground state to the first excited state $\left(\Delta E_{g}\right)$ as a function of symmetric gate charge $\boldsymbol{n}_{g}-\boldsymbol{n}_{g 0}=n_{\text {diag }} \mathbf{1}$ and $\varphi_{\text {diag }}$ for $E_{J} / E_{C}=1.0$. Note that the characteristic scale is now set by $E_{C}$ rather than $E_{J}$. [(c)-(e)] Line cuts of $E_{g}$ (pink) and the Berry curvature $\Omega$ (black) along $\varphi_{\text {diag }}$ for the indicated gate charge values. For presentation purposes, the Berry curvature for each plot is normalized by $\Omega_{0.4}=10|\Omega(0.4 \times 2 \pi)|$.

from the main text, targeting $E_{J}=E_{C} / 2$ with all junctions identical. To simulate disorder, we add random deviations in the junction area $A$, sampling from a Gaussian distribution with a conservative standard deviation of $10 \%$. Note that errors in the junction area affect both $E_{J} \propto A$ and $E_{C} \propto$ $A^{-1}$. For each instance, we conduct a numerical search for spectral nodes in flux space, fixing the gate charge value $\boldsymbol{n}_{g}=\boldsymbol{n}_{g 0}$. We find the topological phase in all 1000 tested instances signaling robustness to experimental fabrication uncertainty.

\section{Noise considerations}

Both flux and charge noise are important to consider in any experimental proposal [84]. For each type, it is convenient to separately consider high-frequency ( $\gtrsim \mathrm{kHz}$ ) and low-frequency ( $\lesssim \mathrm{Hz}$, also known as "drift") parts. High-frequency noise introduces measurable spectroscopic linewidth and reduces phase coherence. For the spectroscopy experiment proposed in Sec. IV, linewidths limit the resolution with which any degeneracies can be determined. The third experiment (direct Berry curvature measurements) will be limited by the relationship between phase coherence time and the measurement time, the determination of which is beyond the scope of our work. Very high frequency noise, at the value of $E_{g}$, will introduce a finite excited-state population. The rate of these unintended transitions sets a lower bound for the speed of the transconductance measurements. In transmons, this rate has been lowered to the $100-\mathrm{Hz}$ range [85], which is five to eight orders of magnitude below anticipated $E_{g}$ scales for the Weyl circuit.

Low-frequency noise, or drift, is a significant problem if it interferes with the typical time-scale of experimental scans. Flux drift is generally insignificant. Charge drift, however, can be large and has typical timescales are of order tens of minutes or more for transmons $[85,86]$. For this reason, it may be sensible to choose $\boldsymbol{k}=\boldsymbol{n}_{g}$ rather than $\boldsymbol{\varphi}_{m}$ for simulation of broken-inversion-symmetry systems, as it may be more convenient to fix the Hamiltonian control parameters for long periods of time. In either case, a solution will be needed if the necessary multidimensional measurement scans are slower than these timescales. This motivates the use of a cQED setup, which can take advantage of fast individual measurements to implement active feedback routines to correct for charge drift (see Ref. [87] for an example of active feedback improving spin qubit coherence).
[1] C. L. Kane and E. J. Mele, Quantum Spin Hall Effect in Graphene, Phys. Rev. Lett. 95, 226801 (2005).

[2] M. Z. Hasan and C. L. Kane, Colloquium: Topological insulators, Rev. Mod. Phys. 82, 3045 (2010).

[3] X.-L. Qi and S.-C. Zhang, Topological insulators and superconductors, Rev. Mod. Phys. 83, 1057 (2011).

[4] R. Leone and L. Lévy, Topological quantization by controlled paths: Application to Cooper pairs pumps, Phys. Rev. B 77, 064524 (2008).
[5] P. Roushan, C. Neill, Y. Chen, M. Kolodrubetz, C. Quintana, N. Leung, M. Fang, R. Barends, B. Campbell, Z. Chen, B. Chiaro, A. Dunsworth, E. Jeffrey, J. Kelly, A. Megrant, J. Mutus, P. J. J. O’Malley, D. Sank, A. Vainsencher, J. Wenner, T. White, A. Polkovnikov, A. N. Cleland, and J. M. Martinis, Observation of topological transitions in interacting quantum circuits, Nature (London) 515, 241 (2014).

[6] L. M. Nash, D. Kleckner, A. Read, V. Vitelli, A. M. Turner, and W. T. M. Irvine, Topological mechanics of 
gyroscopic metamaterials, Proc. Natl. Acad. Sci. USA 112, 14495 (2015).

[7] L. Lu, J. D. Joannopoulos, and M. Soljačić, Topological states in photonic systems, Nat. Phys. 12, 626 (2016).

[8] X. Wan, A. M. Turner, A. Vishwanath, and S. Y. Savrasov, Topological semimetal and Fermi-arc surface states in the electronic structure of pyrochlore iridates, Phys. Rev. B 83, 205101 (2011).

[9] N. P. Armitage, E. J. Mele, and A. Vishwanath, Weyl and Dirac semimetals in three-dimensional solids, Rev. Mod. Phys. 90, 015001 (2018).

[10] R. Yu, H. Weng, Z. Fang, H. Ding, and X. Dai, Determining the chirality of Weyl fermions from circular dichroism spectra in time-dependent angle-resolved photoemission, Phys. Rev. B 93, 205133 (2016).

[11] C.-K. Chan, N. H. Lindner, G. Refael, and P. A. Lee, Photocurrents in Weyl semimetals, Phys. Rev. B 95, 041104(R) (2017).

[12] F. de Juan, A. G. Grushin, T. Morimoto, and J. E. Moore, Quantized circular photogalvanic effect in Weyl semimetals, Nat. Commun. 8, 15995 (2017).

[13] Q. Ma, S.-Y. Xu, C.-K. Chan, C.-L. Zhang, G. Chang, Y. Lin, W. Xie, T. Palacios, H. Lin, S. Jia, P. A. Lee, P. Jarillo-Herrero, and N. Gedik, Direct optical detection of Weyl fermion chirality in a topological semimetal, Nat. Phys. 13, 842 (2017).

[14] Z. Lin and Z. Liu, Spin-1 Dirac-Weyl fermions protected by bipartite symmetry, J. Chem. Phys. 143, 214109 (2015).

[15] B. Bradlyn, J. Cano, Z. Wang, M. G. Vergniory, C. Felser, R. J. Cava, and B. A. Bernevig, Beyond Dirac and Weyl fermions: Unconventional quasiparticles in conventional crystals, Science 353, aaf5037 (2016).

[16] G. Chang, S.-Y. Xu, S.-M. Huang, D. S. Sanchez, C.-H. Hsu, G. Bian, Z.-M. Yu, I. Belopolski, N. Alidoust, H. Zheng, T.-R. Chang, H.-T. Jeng, S. A. Yang, T. Neupert, H. Lin, and M. Z. Hasan, Nexus fermions in topological symmorphic crystalline metals, Sci. Rep. 7, 1688 (2017).

[17] A. Bernevig, H. Weng, Z. Fang, and X. Dai, Recent progress in the study of topological semimetals, J. Phys. Soc. Jpn. 87, 041001 (2018).

[18] L. Lu, Z. Wang, D. Ye, L. Ran, L. Fu, J. D. Joannopoulos, and M. Soljačić, Experimental observation of Weyl points, Science 349, 622 (2015).

[19] D. Z. Rocklin, B. G. Chen, M. Falk, V. Vitelli, and T. C. Lubensky, Mechanical Weyl Modes in Topological Maxwell Lattices, Phys. Rev. Lett. 116, 135503 (2016).

[20] X. Tan, Y. Zhao, Q. Liu, G. Xue, H. Yu, Z. D. Wang, and Y. $\mathrm{Yu}$, Realizing and manipulating space-time inversion symmetric topological semimetal bands with superconducting quantum circuits, npj Quantum Mater. 2, 60 (2017).

[21] T. Zhang, Z. Song, A. Alexandradinata, H. Weng, C. Fang, L. $\mathrm{Lu}$, and Z. Fang, Double-Weyl Phonons in Transition-Metal Monosilicides, Phys. Rev. Lett. 120, 016401 (2018).

[22] Y. Lu, N. Jia, L. Su, C. Owens, G. Juzeliūnas, D. I. Schuster, and J. Simon, Probing the Berry curvature and Fermi arcs of a Weyl circuit, Phys. Rev. B 99, 020302(R) (2019).

[23] I. M. Georgescu, S. Ashhab, and F. Nori, Quantum simulation, Rev. Mod. Phys. 86, 153 (2014).

[24] R. Leone and A. Monjou, Merging diabolical points of a superconducting circuit, Condens. Matter Phys. 16, 33801 (2013).
[25] X. Tan, D.-W. Zhang, Q. Liu, G. Xue, H.-F. Yu, Y.-Q. Zhu, H. Yan, S.-L. Zhu, and Y. Yu, Topological Maxwell Metal Bands in a Superconducting Qutrit, Phys. Rev. Lett. 120, 130503 (2018).

[26] A. Y. Kitaev, Unpaired Majorana fermions in quantum wires, Phys. Usp. 44, 131 (2001).

[27] L. B. Ioffe, M. V. Feigel'man, A. Ioselevich, D. Ivanov, M. Troyer, and G. Blatter, Topologically protected quantum bits using Josephson junction arrays, Nature (London) 415, 503 (2002).

[28] A. Kitaev, Protected qubit based on a superconducting current mirror, arXiv:cond-mat/0609441.

[29] S. Gladchenko, D. Olaya, E. Dupont-Ferrier, B. Douçot, L. B. Ioffe, and M. E. Gershenson, Superconducting nanocircuits for topologically protected qubits, Nat. Phys. 5, 48 (2009).

[30] B. van Heck, S. Mi, and A. R. Akhmerov, Single fermion manipulation via superconducting phase differences in multiterminal Josephson junctions, Phys. Rev. B 90, 155450 (2014).

[31] T. Yokoyama and Y. V. Nazarov, Singularities in the Andreev spectrum of a multiterminal Josephson junction, Phys. Rev. B 92, 155437 (2015).

[32] R.-P. Riwar, M. Houzet, J. S. Meyer, and Y. V. Nazarov, Multi-terminal Josephson junctions as topological matter, Nat. Commun. 7, 11167 (2016).

[33] J. S. Meyer and M. Houzet, Nontrivial Chern Numbers in Three-Terminal Josephson Junctions, Phys. Rev. Lett. 119, 136807 (2017).

[34] L. Peralta Gavensky, G. Usaj, D. Feinberg, and C. A. Balseiro, Berry curvature tomography and realization of topological Haldane model in driven three-terminal Josephson junctions, Phys. Rev. B 97, 220505(R) (2018).

[35] R. L. Klees, G. Rastelli, J. C. Cuevas, and W. Belzig, Microwave Spectroscopy Reveals the Quantum Geometric Tensor of Topological Josephson Matter, Phys. Rev. Lett. 124, 197002 (2020).

[36] Y. Cohen, Y. Ronen, J.-H. Kang, M. Heiblum, D. Feinberg, R. Mélin, and H. Shtrikman, Nonlocal supercurrent of quartets in a three-terminal Josephson junction, Proc. Natl. Acad. Sci. USA 115, 6991 (2018).

[37] A. W. Draelos, M.-T. Wei, A. Seredinski, H. Li, Y. Mehta, K. Watanabe, T. Taniguchi, I. V. Borzenets, F. Amet, and G. Finkelstein, Supercurrent flow in multiterminal graphene Josephson junctions, Nano Lett. 19, 1039 (2019).

[38] G. V. Graziano, J. S. Lee, M. Pendharkar, C. J. Palmstrøm, and V. S. Pribiag, Transport studies in a gate-tunable three-terminal Josephson junction, Phys. Rev. B 101, 054510 (2020).

[39] N. Pankratova, H. Lee, R. Kuzmin, K. Wickramasinghe, W. Mayer, J. Yuan, M. G. Vavilov, J. Shabani, and V. E. Manucharyan, Multiterminal Josephson Effect, Phys. Rev. X 10, 031051 (2020).

[40] S. Imhof, C. Berger, F. Bayer, J. Brehm, L. W. Molenkamp, T. Kiessling, F. Schindler, C. H. Lee, M. Greiter, T. Neupert, and R. Thomale, Topolectrical-circuit realization of topological corner modes, Nat. Phys. 14, 925 (2018).

[41] E. Zhao, Topological circuits of inductors and capacitors, Ann. Phys. 399, 289 (2018).

[42] U. Vool and M. Devoret, Introduction to quantum electromagnetic circuits, Int. J. Circuit Theory Appl. 45, 897 (2017).

[43] D. A. Ivanov, L. B. Ioffe, V. B. Geshkenbein, and G. Blatter, Interference effects in isolated Josephson junction arrays with geometric symmetries, Phys. Rev. B 65, 024509 (2001). 
[44] J.-P. Hanke, F. Freimuth, C. Niu, S. Blügel, and Y. Mokrousov, Mixed Weyl semimetals and low-dissipation magnetization control in insulators by spin-orbit torques, Nat. Commun. 8, 1479 (2017).

[45] P. Kotetes, M. T. Mercaldo, and M. Cuoco, Synthetic Weyl Points and Chiral Anomaly in Majorana Devices with Nonstandard Andreev-Bound-State Spectra, Phys. Rev. Lett. 123, 126802 (2019).

[46] We note that this circuit was previously inspected [78] in a strongly symmetry-dependent context (e.g., all Josephson and charging energies must be equal) in order to generate a quadratically protected degeneracy at the point where the circuit is tuned so that six charge states are equal in energy. We are unaware of prior work pointing out the existence of Weyl nodes in this circuit.

[47] The triply degenerate point appears to realize an effective spin-1 system predicted in Ref. [15]. We do not inspect it in detail due to its sensitivity to symmetry and the need to tune to a particular offset charge point. It was also seen in a multiterminal Andreev proposal [73].

[48] A. Akhmerov, Connecting the dots, https://quantumtinkerer. tudelft.nl/blog/connecting-the-dots/.

[49] I. Belopolski, P. Yu, D. S. Sanchez, Y. Ishida, T.-R. Chang, S. S. Zhang, S.-Y. Xu, D. Mou, H. Zheng, G. Chang, G. Bian, H.-T. Jeng, T. Kondo, A. Kaminski, H. Lin, Z. Liu, S. Shin, and M. Z. Hasan, A minimal, "hydrogen atom" version of an inversionbreaking Weyl semimetal, arXiv:1610.02013.

[50] V. Gritsev and A. Polkovnikov, Dynamical quantum Hall effect in the parameter space, Proc. Natl. Acad. Sci. USA 109, 6457 (2012).

[51] A. Wallraff, D. I. Schuster, A. Blais, L. Frunzio, R.-S. Huang, J. Majer, S. Kumar, S. M. Girvin, and R. J. Schoelkopf, Strong coupling of a single photon to a superconducting qubit using circuit quantum electrodynamics, Nature (London) 431, 162 (2004).

[52] Y. Kubo, F. R. Ong, P. Bertet, D. Vion, V. Jacques, D. Zheng, A. Dréau, J.-F. Roch, A. Auffeves, F. Jelezko, J. Wrachtrup, M. F. Barthe, P. Bergonzo, and D. Esteve, Strong Coupling of a Spin Ensemble to a Superconducting Resonator, Phys. Rev. Lett. 105, 140502 (2010).

[53] C. Janvier, L. Tosi, L. Bretheau, C. O. Girit, M. Stern, P. Bertet, P. Joyez, D. Vion, D. Esteve, M. F. Goffman, H. Pothier, and C. Urbina, Coherent manipulation of Andreev states in superconducting atomic contacts, Science 349, 1199 (2015).

[54] L. Tosi, C. Metzger, M. F. Goffman, C. Urbina, H. Pothier, S. Park, A. L. Yeyati, J. Nygård, and P. Krogstrup, Spin-Orbit Splitting of Andreev States Revealed by Microwave Spectroscopy, Phys. Rev. X 9, 011010 (2019).

[55] J. Edstam and H. K. Olsson, Josephson broadband spectroscopy to $1 \mathrm{THz}$, Appl. Phys. Lett. 64, 2733 (1994).

[56] J. Leppäkangas, E. Thuneberg, R. Lindell, and P. Hakonen, Tunneling of Cooper pairs across voltage-biased asymmetric single-Cooper-pair transistors, Phys. Rev. B 74, 054504 (2006).

[57] P.-M. Billangeon, F. Pierre, H. Bouchiat, and R. Deblock, Very High Frequency Spectroscopy and Tuning of a Single-CooperPair Transistor with an On-Chip Generator, Phys. Rev. Lett. 98, 126802 (2007).

[58] L. Bretheau, C. O. Girit, H. Pothier, D. Esteve, and C. Urbina, Exciting Andreev pairs in a superconducting atomic contact, Nature (London) 499, 312 (2013).
[59] L. Bretheau, C. O. Girit, C. Urbina, D. Esteve, and H. Pothier, Supercurrent Spectroscopy of Andreev States, Phys. Rev. X 3, 041034 (2013).

[60] E. Eriksson, R.-P. Riwar, M. Houzet, J. S. Meyer, and Y. V. Nazarov, Topological transconductance quantization in a fourterminal Josephson junction, Phys. Rev. B 95, 075417 (2017).

[61] E. V. Repin, Y. Chen, and Y. V. Nazarov, Topological properties of multiterminal superconducting nanostructures: Effect of a continuous spectrum, Phys. Rev. B 99, 165414 (2019).

[62] L. J. Geerligs, S. M. Verbrugh, P. Hadley, J. E. Mooij, H. Pothier, P. Lafarge, C. Urbina, D. Estève, and M. H. Devoret, Single Cooper pair pump, Z. Phys. B Condens. Matter 85, 349 (1991).

[63] L. Peyruchat, J. Griesmar, J.-D. Pillet, and Ç. Ö. Girit, Transconductance quantization in a topological Josephson tunnel junction circuit, Phys. Rev. Research 3, 013289 (2021).

[64] N.-H. Kaneko, S. Nakamura, and Y. Okazaki, A review of the quantum current standard, Meas. Sci. Technol. 27, 032001 (2016).

[65] M. Kolodrubetz, D. Sels, P. Mehta, and A. Polkovnikov, Geometry and non-adiabatic response in quantum and classical systems, Phys. Rep.Geom. Non-Adiabatic Resp. Quantum Class. Syst. 697, 1 (2017).

[66] M. D. Schroer, M. H. Kolodrubetz, W. F. Kindel, M. Sandberg, J. Gao, M. R. Vissers, D. P. Pappas, A. Polkovnikov, and K. W. Lehnert, Measuring a Topological Transition in an Artificial Spin-1/2 System, Phys. Rev. Lett. 113, 050402 (2014).

[67] C. L. Kane and E. J. Mele, $Z_{2}$ Topological Order and the Quantum Spin Hall Effect, Phys. Rev. Lett. 95, 146802 (2005).

[68] M. Mirrahimi, Z. Leghtas, V. V. Albert, S. Touzard, R. J. Schoelkopf, L. Jiang, and M. H. Devoret, Dynamically protected cat-qubits: A new paradigm for universal quantum computation, New J. Phys. 16, 045014 (2014).

[69] Z. Leghtas, S. Touzard, I. M. Pop, A. Kou, B. Vlastakis, A. Petrenko, K. M. Sliwa, A. Narla, S. Shankar, M. J. Hatridge, M. Reagor, L. Frunzio, R. J. Schoelkopf, M. Mirrahimi, and M. H. Devoret, Confining the state of light to a quantum manifold by engineered two-photon loss, Science 347, 853 (2015).

[70] U. D. Giovannini and H. Hübener, Floquet analysis of excitations in materials, J. Phys.: Mater. 3, 012001 (2019).

[71] H. Z. Jooya, K. Reihani, and S.-I. Chu, A graph-theoretical representation of multiphoton resonance processes in superconducting quantum circuits, Sci. Rep. 6, 37544 (2016).

[72] M. Houzet and J. S. Meyer, Majorana-Weyl crossings in topological multiterminal junctions, Phys. Rev. B 100, 014521 (2019).

[73] L. Peralta Gavensky, G. Usaj, and C. A. Balseiro, Topological phase diagram of a three-terminal Josephson junction: From the conventional to the Majorana regime, Phys. Rev. B 100, 014514 (2019).

[74] J. S. Meyer and M. Houzet, Conductance quantization in topological Josephson trijunctions, arXiv:1911.07705.

[75] E. V. Repin and Y. V. Nazarov, Weyl points in the multiterminal hybrid superconductor-semiconductor nanowire devices, arXiv:2010.11494.

[76] K. Sakurai, M. T. Mercaldo, S. Kobayashi, A. Yamakage, S. Ikegaya, T. Habe, P. Kotetes, M. Cuoco, and Y. Asano, Nodal Andreev spectra in multi-Majorana three-terminal Josephson junctions, Phys. Rev. B 101, 174506 (2020). 
[77] V. Fatemi, A. Akhmerov, and L. Bretheau, Weyl Josephson circuits data (2020), Zenodo, http://doi.org/10.5281/zenodo. 4008550

[78] M. V. Feigel'man, L. B. Ioffe, V. B. Geshkenbein, P. Dayal, and G. Blatter, Superconducting Tetrahedral Quantum Bits, Phys. Rev. Lett. 92, 098301 (2004).

[79] C. W. Groth, M. Wimmer, A. R. Akhmerov, and X. Waintal, Kwant: A software package for quantum transport, New J. Phys. 16, 063065 (2014).

[80] T. P. Orlando, J. E. Mooij, L. Tian, C. H. van der Wal, L. S. Levitov, S. Lloyd, and J. J. Mazo, Superconducting persistentcurrent qubit, Phys. Rev. B 60, 15398 (1999).

[81] J. Koch, T. M. Yu, J. Gambetta, A. A. Houck, D. I. Schuster, J. Majer, A. Blais, M. H. Devoret, S. M. Girvin, and R. J. Schoelkopf, Charge-insensitive qubit design derived from the Cooper pair box, Phys. Rev. A 76, 042319 (2007).

[82] R. Leone, L. P. Lévy, and P. Lafarge, Cooper-Pair Pump as a Quantized Current Source, Phys. Rev. Lett. 100, 117001 (2008).
[83] J. M. Kreikebaum, K. P. O’Brien, A. Morvan, and I. Siddiqi, Improving wafer-scale Josephson junction resistance variation in superconducting quantum coherent circuits, Supercond. Sci. Technol. 33, 06LT02 (2020).

[84] P. Krantz, M. Kjaergaard, F. Yan, T. P. Orlando, S. Gustavsson, and W. D. Oliver, A quantum engineer's guide to superconducting qubits, Appl. Phys. Rev. 6, 021318 (2019).

[85] K. Serniak, S. Diamond, M. Hays, V. Fatemi, S. Shankar, L. Frunzio, R. J. Schoelkopf, and M. H. Devoret, Direct Dispersive Monitoring of Charge Parity in Offset-Charge-Sensitive Transmons, Phys. Rev. Appl. 12, 014052 (2019).

[86] B. G. Christensen, C. D. Wilen, A. Opremcak, J. Nelson, F. Schlenker, C. H. Zimonick, L. Faoro, L. B. Ioffe, Y. J. Rosen, J. L. DuBois, B. L. T. Plourde, and R. McDermott, Anomalous charge noise in superconducting qubits, Phys. Rev. B 100, 140503(R) (2019).

[87] M. D. Shulman, S. P. Harvey, J. M. Nichol, S. D. Bartlett, A. C. Doherty, V. Umansky, and A. Yacoby, Suppressing qubit dephasing using real-time Hamiltonian estimation, Nat. Commun. 5, 5156 (2014). 